\title{
ON CERTAIN CYCLES IN GRAPHS
}

\author{
by DOUGLAS D. GRANT
}

(Received 26th June 1979)

\section{Introduction}

We show that every simple graph of order $2 r$ and minimum degree $\geqq 4 r / 3$ has the property that for any partition of its vertex set into 2 -subsets, there is a cycle which contains exactly one vertex from each 2 -subset. We show that the bound $4 r / 3$ cannot be lowered to $r$, but conjecture that it can be lowered to $r+1$.

\section{Definitions}

Throughout this paper $n$ will denote a positive integer and $r$ an integer $\geqq 3$. Given a real number $x,\lceil x\rceil$ will denote the least integer $\geqq x$. Our basic graph-theoretic terminology and notation is that of Bondy and Murty (2), save that we use the word "graph" to mean "simple graph".

We shall require the following notation. Let $G$ be a graph, let $\xi, \eta \in V(G)$, let $W \subset V(G)$ and let $H \subset G$. Then $\tilde{W}$ denotes $V(G) \backslash W, \tilde{H}$ denotes $G[V(G) \backslash V(H)]$, $e(\xi, W)$ denotes the number of edges incident with $\xi$ whose other end lies in $W, e(\xi, \eta)$ denotes $e(\xi,\{\eta\})$ and $W \delta$ denotes the set of edges exactly one of whose ends lies in $W$. If $\xi \in V(H)$, then the $H$-degree of $\xi$ is the degree of $\xi$ in the graph $H$.

Let $G$ be a graph of order $n r$ and let $\Pi=\left\{V_{1}, V_{2}, \ldots, V_{r}\right\}$ be a partition of $V(G)$ into $n$-subsets. We say that $I$ is an $n$-partition of $G$. If $H$ is a cycle of $G$ such that $\left|V(H) \cap V_{i}\right|=1$ for $i=1,2, \ldots, r$, then we say that $H$ is a $\Pi$-cycle of $G$. If there exists a $\Pi$-cycle of $G$, then we say that $G$ is $\Pi$-round. If $G$ is $\Pi$-round for all $n$-partitions $\Pi$ of $G$, then we say that $G$ is $n$-round.

Our aim is to seek bounds for the least integer $p=h(n, r)$ so that if $|V(G)|=n r$ and $\delta(G) \geqq p$, then $G$ is $n$-round. Most of our attention will be devoted to the case $n=2$.

Since a graph is 1-round if and only if it has a hamiltonian cycle, a well-known theorem of Dirac (4) determines that $h(1, r)=\lceil r / 2\rceil$. An unpublished result of Graver (see (1)) implies that $h(n, 3)=2 n$. In the next section we shall consider the function $h(2, r)$.

\section{2-round graphs}

The theorem of Dirac mentioned in Section 2 implies that $h(2, r) \leqq\lceil 3 r / 2\rceil ;$ if $|V(G)|=2 r$ and $\delta(G) \geqq 3 r / 2$, then any $r$ vertices of $G$ induce a subgraph with minimum degree $\geqq r / 2$, which has, by Dirac's Theorem, a hamiltonian cycle. If $G_{r}$ is the union of two complete graphs of order $r+1$ which share just two vertices $\xi$ and $\eta$, then $G_{r}$ is not 
$\Pi$-round for any 2-partition $\Pi$ of $G_{r}$ which contains $\{\xi, \eta\}$. Since $\delta\left(G_{r}\right)=r$, this implies $h(2, r) \geqq r+1$. We conjecture that in fact $h(2, r)=r+1$. In the remainder of this section we prove that $h(2, r) \leqq\lceil 4 r / 3\rceil$.

Theorem 1. If $|V(G)|=2 r$, $\Pi$ is a 2-partition of $G$ and $d(\xi)+d(\eta) \geqq(8 r-1) / 3$ whenever $\{\xi, \eta\} \in \Pi$, then $G$ is $\Pi$-round.

Proof. For each $\xi \in V(G)$, let $\xi^{\prime}$ denote the other vertex of $G$ in the same cell of $I$ as $\xi$. Let $W$ be a transversal of $\Pi$ such that $|W \delta|$ is as small as possible and let $H=G[W]$. Let $\xi \in W$. By the minimality of $|W \delta|$,

$$
\begin{aligned}
0 & \leqq\left|\left[(W \backslash\{\xi\}) \cup\left\{\xi^{\prime}\right\}\right] \delta\right|-|W \delta| \\
& =\left[e(\xi, W)+e\left(\xi^{\prime}, \tilde{W}\right)+e\left(\xi, \xi^{\prime}\right)\right]-\left[e(\xi, \tilde{W})+e\left(\xi^{\prime}, W\right)-e\left(\xi, \xi^{\prime}\right)\right] \\
& =e(\xi, W)+e\left(\xi^{\prime}, \tilde{W}\right)+2 e\left(\xi, \xi^{\prime}\right)-\left[d(\xi)-e(\xi, W)+d\left(\xi^{\prime}\right)-e\left(\xi^{\prime}, \tilde{W}\right)\right] \\
& =2\left[e(\xi, W)+e\left(\xi^{\prime}, \tilde{W}\right)+e\left(\xi, \xi^{\prime}\right)\right]-\left[d(\xi)+d\left(\xi^{\prime}\right)\right] \\
& \leqq 2\left[e(\xi, W)+e\left(\xi^{\prime}, \tilde{W}\right)+e\left(\xi, \xi^{\prime}\right)\right]-(8 r-1) / 3
\end{aligned}
$$

and so

$$
e(\xi, W)+e\left(\xi^{\prime}, \tilde{W}\right) \geqq(8 r-1) / 6-e\left(\xi, \xi^{\prime}\right) \geqq(8 r-7) / 6 \text {. }
$$

Hence

$$
d_{\mathbf{H}}(\xi)+d_{\tilde{H}}\left(\xi^{\prime}\right) \geqq(8 r-7) / 6 .
$$

Since $\xi$ was an arbitrary vertex in $W$, it follows that there are either $\geqq r / 2$ vertices of $H$-degree $\geqq(8 r-7) / 12$ or $\geqq r / 2$ vertices of $\tilde{H}$-degree $\geqq(8 r-7) / 12$. In addition, from (1) it follows that $\delta(H) \geqq(2 r-1) / 6$ and $\delta(\tilde{H}) \geqq(2 r-1) / 6$. By a theorem of Chvátal (3) it follows that either $H$ or $\tilde{H}$ has a hamiltonian cycle, which is a $\Pi$-cycle of $G$. Hence $G$ is $\Pi$-round.

Corollary 2. $h(2, r) \leqq\lceil 4 r / 3\rceil$.

We now know that $r+1 \leqq h(2, r) \leqq\lceil 4 r / 3\rceil$. The upper and lower bounds coincide for $r=3$. We can show that $h(2,4)=5$ and $h(2,5)=6$, confirming our conjecture and improving on Corollary 2 in the cases $r=4$ and 5.

\section{Application to polar graphs}

In a series of papers (5), (6), (7), (8) Zelinka introduced to the literature the concepts of polar graphs and polarised graphs first defined by F. Zitek. The results of Section 3 can be interpreted in the context of hamiltonian homopolar cycles in polar graphs. The definitions relevant to the present section can be found in the papers of Zelinka.

Let $\theta(r)$ be the least integer $y$ so that if $P$ is a polar graph of order $r$ each of whose poles has degree (see $(8)$ ) $\geqq y$, then $P$ has a hamiltonian homopolar (see (6)) cycle.

Proposition 3. $\theta(r)=h(2, r)-1$.

Sketch of proof. Given a graph $G$ of order $2 r$ with a 2-partition $\Pi=\left\{\left\{\xi_{1}, \eta_{1}\right\}\right.$, $\left.\left\{\xi_{2}, \eta_{2}\right\}, \ldots,\left\{\xi_{r}, \eta_{r}\right\}\right\}$, form a polar graph $P(G, \Pi)$ by merging each $\xi_{i}$ and $\eta_{i}$ into one 
vertex $\zeta_{i}$ of $P(G, \Pi)$, assigning incidences of edges with $\xi_{i}$ to one pole of $\zeta_{i}$ and incidences with $\eta_{i}$ to the other pole of $\zeta_{i}$. This construction can be reversed, to produce from a polar graph $P$ a graph $G(P)$ together with a 2-partition $\Pi(P)$ of $G(P)$, where for the purposes of the present investigation we insist on the vertices in a cell of $\Pi(P)$ being adjacent.

A $\Pi$-cycle of $G$ corresponds to a hamiltonian homopolar cycle in $P(G, \Pi)$, and a hamiltonian homopolar cycle in $P$ corresponds to a $\Pi(P)$-cycle in $G(P)$. By means of this correspondence it follows that $\theta(r)=h(2, r)-1$.

Corollary 4. $r \leqq \theta(r) \leqq\lceil 4 r / 3\rceil-1$.

To close we remark that the conjecture of Section 3 is equivalent to $\theta(r)=r$.

Acknowledgement. I would like to thank Professor C. St. J. A. Nash-Williams, University of Reading, who supervised my Ph.D. Thesis, in which the material of this paper appears.

\section{REFERENCES}

(1) B. Bollobás, P. Erdös and E. Szénreredi, On Complete Subgraphs of $r$-chromatic Graphs, Discrete Math. 13 (1975), 97-107.

(2) J. A. Bondy and U. S. R. Murty, Graph Theory with Applications, (Macmillan, London and Basingstoke, 1976).

(3) V. Chvátal, On Hamilton's Ideals, J. Combinatorial Theory 12B (1972), 163-168.

(4) G. A. Dirac, Some Theorems on Abstract Graphs, Proc. London Math. Soc. 2 (1952), 69-81.

(5) B. Zelinka, Isomorphisms of Polar and Polarised Graphs, Czech. Math. J. 26 (1976), 339-351.

(6) B. Zelinka, Analoga of Menger's Theorem for Polar and Polarised Graphs, Czech. Math. J. 26 (1976), 352-360.

(7) B. Zelinka, Eulerian Polar Graphs, Czech. Math. J. 26 (1976), 361-364.

(8) B. Zelinka, Self-derived Polar Graphs, Czech. Math. J. 26 (1976), 365-370.

Napier College

EDINBURGH 